\title{
Methode zur direkten Methylierung dünnschichtchromatographisch aufgetrennter Lipide ohne deren Elution aus Kieselgel
}

\author{
Von P. HuŠEK \\ Aus dem Forschungsinstitut für Endokrinologie in Prag (Direktor: Doc. Dr. K. Šilink)
}

(Eingegangen am 29. Juli 1969)

Für die schnelle Überführung der dünnschichtchromatographisch aufgetrennten Lipide in die für gaschromatographische Analyse erforderlichen Fettsäuremethylester wird hier eine einfache und quantitative Methode beschrieben, die ohne Elution der Lipide aus Kieselgel die Methylierung innerhalb einiger Minuten gewährleistet.

\section{A method for the direct methylation of lipids separated by thin layer cbromatograpby without elution from the silicagel}

A simple quantitative method is described for the direct methylation of lipids separated by thin layer chromatography without elution from the silicagel. The conversion takes a few minutes and the resulting fatty acid methyl esters may then be submitted to gas chromatography.

In der letzten Zeit wurde immer häufiger versucht, die Analyse der Lipide ohne vorherige Veränderung der chemischen Struktur direkt gaschromatographisch durchzuführen $(1,2)$. Sie sind aber wegen ihrer geringen Flüchtigkeit als Folge des hohen Molekulargewichtes, wegen der hohen Polarität und der thermischen Labilität mancher ihrer Fraktionen für die gaschromatographische Bestimmung nicht sehr geeignet. Zum anderen wird die Trennschärfe und somit auch die analytische Bestimmung durch die erforderliche niedrige Konzentration der stationären Phase beeinträchtigt, darüber hinaus muß man sowohl hohe Arbeitstemperaturen (programmiert bis $z u 4^{\circ}$ ) als auch thermisch resistente stationäre Phasen wie z. B. SE-30, OV-1 oder JXR verwenden.

Der Ausbau der Methodik zur direkten Bestimmung der Lipide wird sicher zu weiteren Fortschritten führen. Die Schwierigkeiten bei der Bestimmung der hochpolaren und thermolabilen Phospholipide scheinen dabei einer Lösung nicht näher zu kommen. Dies bedingt die gegenwärtig starken Bemühungen, die am häufigsten verwandte Methodik - Methylester-Bildung der Fettsäuren - zu verbessern.

$\mathrm{Da}$ alle Lipidgruppen dieselben Methylester bilden, ist eine vorherige dünnschichtchromatographische Trennung in die Einzelfraktionen erforderlich. Die hohe Polarität der Phospholipide ist hier genauso wie bei der Gaschromatographie das schwierigste Problem, wobei eine 100proz. Rückgewinnung durch Elution aus Kieselgel praktisch unmöglich ist $(3,4,5)$. Nach unserer heutigen Kenntnis verwendet man folgende Methodik: Während zur Elution aller anderen Lipidfraktionen relativ kleine Mengen von niedrigsiedenden Lösungsmitteln (Dichlormethan, Diäthyläther, Chloroform) ausreichen, benötigt die Phospholipid-Fraktion eine bis zehnfache Menge von weniger flüchtigen Elutionsmitteln, die meistens aus verschiedenen Gemischen von Chloroform, Methylalkohol und Wasser bestehen. Die schwierige Elution der Phosphatide mit der erforderlichen, zeitraubenden Elutionsmittelverdampfung verzögert so das ganze Verfahren der kompletten Lipidbestimmung, so daß die Vorteile der Anwendung der Dünnschichtchromatographie beeinträchtigt werden.

Diese Tatsache war Anlaß zu einer Reihe von Versuchen, die Elution zu umgehen. Die hier beschriebene Methodik faßt die Elution mit alkalisch-katalysierter Umesterung zusammen, wodurch die Elutionszeit und die sonst erforderlichen Elutionsmittel eingespart werden. Die Methode wurde ursprünglich für Phospholipide entwickelt, hat sich aber auch für andere Lipidfraktionen bewährt. Die alkalisch-katalysierte Umesterung mit NaMethylat knüpft an die ursprüngliche Arbeit von LuDDY (6) und besonders an die in der Veröffentlichung von Doss und Oetre (7) enthaltenen Erfahrungen an.

\section{Methodik}

\section{Lipidextraktion}

Zur Lipidextraktion benötigt man gewöhnlich 0,1 bis $1,0 \mathrm{~m} l$ Serum. Das Extraktionsverfahren kann man entweder nach FolCh und Mitarbeitern $(8)$ oder nach ReNkoNen $(9,10)$ durchführen. Die erste Methodik ist wirksam und einfach, nimmt jedoch 24 Stdn. Zeit in Anspruch, dagegen verkürzt die zweite die Arbeitszeit auf etwa 1 Std., die Behandlung ist aber anspruchsvoller und die Ergebnisse sind bestenfalls denen von FoLCH gleich. Bei der hier vorliegenden Untersuchung wurde die FoLcHExtraktionsmethode angewandt.

\section{Dünnschichtchromatographie}

Zur Auftrennung der Lipide kann man verschiedene KieselgelSorten benutzen. Kieselgel wird dabei normalerweise auf Platten $20 \times 20 \mathrm{~cm}$ oder $5 \times 20 \mathrm{~cm}$ aufgetragen, wobei die Schichtdicke 0,1 bis $0,5 \mathrm{~mm}$ für „geklebte" Platten und 1,0 bis $2,0 \mathrm{~mm}$ für „gestreute“ Platten beträgt. Man wählt zweckmäßigerwcise solche Bedingungen, bei denen eine vollständige Auftrennung der Lipide in einem Lauf erzielt wird. Hier hat sich das Lösungs-

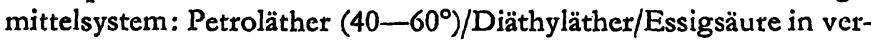
schiedenen Mischungsverhältnissen $(84-79: 15-20: 1 \mathrm{v} / \mathrm{v})$ bewährt. 
Um die universelle Anwendbarkeit der hier beschriebenen Methode für verschiedene Kieselgel-Sorten zu überprüfen, wurde die dünnschichtchromatographische Trennung mit folgenden drei Sorten von Kieselgel ${ }^{1}$ ) durchgeführt:

a) Kieselgel HR reinst, $10-50 \mu \mathrm{m}$, ,geklebte“ Platten bis zu $0,5 \mathrm{~mm}$ Schichtdicke.

b) Kieselgel $\mathrm{GF}_{254}$ (Gipszusatz $13 \%$ ), $10-40 \mu \mathrm{m}$, „geklebte“ Platten bis zu $0,5 \mathrm{~mm}$ Schichtdicke.

c) Kieselgel 0,05-0,2 mm ( $70-325$ mesh), „gestreute“ Platten von 1 bis $2 \mathrm{~mm}$ Schichtdicke.

Die beiden ersten Sorten wurden jeweils im Verhältnis $1: 2(\mathrm{~g} / \mathrm{g})$ mit Wasser versetzt, dann mit einem Streichgerät auf den Platten zur erforderlichen Schichtdicke ausgestrichen und nach Lufttrocknung $1 \mathrm{Std}$. lang bei $110^{\circ}$ aktiviert. Die Entwicklung erfolgte in nahezu senkrechter Lage der Platten.

Die dritte Kieselgel-Sorte wurde auf die Platte gestreut und mit Glasstäbchen zu einer Schicht von etwa $1,5 \mathrm{~mm}$ Dicke ausgestrichen. Die Entwicklung wurde hier in einem Winkel von $30^{\circ}$ durchgeführt.

Die mit dem Lösungsmittelsystem Petroläther/Diathyläther/ Essigsäure (81:18:1 v/v) durchgeführte Auftrennung war bei Kieselgel HR und $\mathrm{GF}_{254}$ Platten ausgezeichnet, bei der dritten Kieselgel-Sorte nur befriedigend.

Die aufgetrennten Lipidfraktionen wurden unter UV-Licht oder mit Hilfe des Sprühreagenz $2^{\prime}, 7^{\prime}$-Dichlorfluorescein (0,1proz. Lösung in Äthanol) sichtbar gemacht. Die markierten KieselgelAbschnitte mit den enthaltenen Lipiden wurden abgesaugt und quantitativ in Reagenzgläser überführt.

Beschreibung des Verfahrens

\section{Reagenzien ${ }^{2}$ )}

$0,5 \mathrm{~N} \mathrm{Na}$-Methylat (wasserfrei: metallisches Natrium in Methanol p. a. unter Kühlung stückweise hineingeben oder die erforderliche Menge von Natriummethylat ${ }^{3}$ ) im Methanol lösen; wasserhaltig: Natriumhydroxyd Plätzchenform im Methanol lösen);

etwa $1,5 \mathrm{~N} \quad \mathrm{BF}_{3}$-Methanol Lösung $(100 \mathrm{~m} l$ von BortrifluoridÄthyläther-Komplex mit $400 \mathrm{ml}$ Methanol mischen);

$0,5 \mathrm{~N}$ wäßr. Salzsäure; Petroleumbenzin (Petroläther) $40-60^{\circ}$; Aceton; Natriumsulfat wasserfrei.

\section{Apparative Ausrüstung}

Reaktionsgefäß $\mathrm{e}^{4}$ ) (Normalschliff 19/26), $15 \mathrm{~cm}$ lang, mit Stopfen, je 2 Stück zur Aufarbeitung der dünnschichtchromatographisch aufgetrennten Lipidgruppe.

\section{Arbeitsvorscbrift}

\section{a) Veresterung der freien Fettsäuren}

$\mathrm{Zu}$ dem abgesaugten Kieselgel mit der Lipidfraktion von freien Fettsäuren werden in das Reagenzglas $4 \mathrm{~m} l \mathrm{BF}_{3}$-Methanol-Lösung gegeben. Die Probe wird $5 \mathrm{Min}$. im Wasserbad auf einer Temperatur von $60^{\circ}$ gehalten. Gleich danach wird das Gemisch im Reaktionsgefäß mit Schliffstoffen bei Raumtemperatur 1-2 Min. kräftig geschüttelt. Nach der Zugabe von $5 \mathrm{ml}$ Petroläther und $10 \mathrm{~m} l$ dest. Wasser erfolgt die Extraktion der Fettsäuremethylester (siehe unten).

Bemerkung: Die Fraktion der freien Fettsäuren kann auch nach folgender Methode behandelt werden: zum abgesaugten Kieselgel werden $5 \mathrm{ml}$ Petroläther und $2 \mathrm{ml}$ Aceton gegeben. Nach 2 Min. Schütteln gibt $\operatorname{man} 5 \mathrm{ml}$ folgender Mischung zu: $22,7 \mathrm{~g}$ $\mathrm{Na}_{2} \mathrm{SO}_{4} \cdot 10 \mathrm{H}_{2} \mathrm{O}, 2 \mathrm{ml} 0,1 \mathrm{~N} \mathrm{H}_{2} \mathrm{SO}_{4}$ aufgefüllt auf $1000 \mathrm{ml}$ dest. Wasser. Dann wird wieder 1 Min. geschüttelt. Die obere organische Phase wird abpipettiert und in das andere Reagenzglas überführt, danach mit je $5 \mathrm{ml}$ dest. Wasser gewaschen. Anschließend wird der Extrakt mit $2 \mathrm{ml}$ ätherischer DiazomethanLösung versetzt und durch Zugabe einer kleinen Menge Natriumsulfat getrocknet. Nach Entfernung des Natriumsulfats durch

\footnotetext{
1) Hersteller: Fa. E. Merck, Darmstadt.

2) Fa. E. Merck, Darmstadt.

3) Fa. EGA-Chemie KG, Steinheim.

$\left.{ }^{4}\right)$ Fa. Quickfit, England.
}

Umgießen wird das Lösungsmittel bei $40^{\circ}$ im Wasserstrahlpumpenvakuum eingedampft.

b) Umesterung von Triglyceriden, Phospholipiden und Cholesterolestern

Zum Kieselgel mit entsprechender Lipidfraktion gibt man in das Reagenzglas $1 \mathrm{ml}$ Petroläther, $1 \mathrm{ml}$ Aceton und $4 \mathrm{ml} 0,5 \mathrm{~N} \mathrm{Na}$ Methylat bei Triglyceriden und Cholesterolestern bzw. $5 \mathrm{~m} /$ Methylat bei Phospholipiden. Die Umesterung der Triglyceride und Phospholipide in die Methylesterform wird durch kräftiges, 1-2 Min. dauerndes Schütteln bei Zimmertemperatur durchgeführt. Die Cholesterolester muß man dagegen, wie die freien Fettsäuren, $5 \mathrm{Min}$. im Wasserbad bei $60^{\circ}$ halten, bevor diese weiter durch 1-2 Min. Schütteln methyliert werden. Nach vollendeter Umesterung wird, gleich bei allen drei Lipidfraktionen, in die Reagenzgläser $5 \mathrm{ml}$ Petroläther und $10 \mathrm{ml} 0,5 \mathrm{~N} w a ̈ ß r . ~ H C l$ gegeben und das weitere Verfahren, das auch die Behandlung der freien Fettsäuren betrifft, wird wie folgt durchgeführt:

c) Extraktion der Fettsäuremethylester:

Das Dreiphasensystem - organiscche Phase, wäßr. Phase und Kieselgel - wird etwa $1 / 2$ Min. so durchgeschüttelt, daß die unterste Kieselgelphase in das ganze Extraktionsvolumen verteilt wird. Nur unter dieser Bedingung erfolgt die Extraktion quantitativ. Nach der Abtrennung der Phasen wird Kieselgel normalerweise sedimentieren, was man durch die Drehung des Reagenzglases um die senkrechte Achse beschleunigen kann (im Falle der höheren Konzentration der Phospholipid-Fraktion kann ein Teil des Kieselgels zwischen den beiden Phasen verbleiben, was die Quantität ungünstig beeinflussen kann; dann sollte das Kieselgel fein abzentrifugiert werden). Mit Hilfe eines zu diesem Zweck angepaßten Pipettiersystems - Injektionsspritze und Pipette sind mit Polyäthylenschlauch verbunden und auf einem Stativ befestigt - wird die obere organischen Phase abgezogen und in das andere Reagenzglas überführt; die untere wäßr. Phase mit Kieselgel wird verworfen. Die abgezogene organische Phase wird zunächst mit $10 \mathrm{ml}$, dann mit $5 \mathrm{ml}$ dest. Wasser durchgeschüttelt und die wäßr. Phasen werden ebenfalls von unten abgezogen und verworfen. Die organische Phase wird mit $\mathrm{Na}_{2} \mathrm{SO}_{4}$ siccum getrocknet, $15 \mathrm{Min}$. stehengelassen und in geeigneten Gefäßen eingedampft. Die Probe ist für gaschromatographische Analyse vorbereitet.

Bemerkungen: $\mathrm{Da}$ Aceton und Petroläther im Wasserbad bei $60^{\circ}$ sieden, sollte man die Stopfen nur leicht aufsetzen, weil sie wegen des Überdruckes beweglich bleiben müßten.

Um die organische Phase nach der ersten Extraktion vollständig abzuziehen, empfiehlt es sich zugleich auch einen kleinen Teil der wäßr. Phase mit abzuziehen (zuletzt die Pipettenspitze an die Berührungsfläche beider Phasen setzen);

Die Reihenfolge der zugegebenen Reagenzien sollte wie beschrieben eingehalten werden, die Begründung findet sich in der Diskussion.

$\mathrm{Da}_{\mathrm{a}}$ freies Cholesterol, das durch die Umesterung der Cholesterolester freigesetzt wird und in der organischen Phase ebenfalls enthalten ist, die gaschromatographische Analyse nicht beeinträchtigt (7), wurde es vorher nicht abgetrennt. Bei nicht zu großen Konzentrationen der Cholesterolester-Fraktion wird die Lebensdauer der gaschromatographischen Säule nicht merklich verkürzt.

\section{Gaschromatographische Analyse}

Am meisten benutzte Kombination stationäre Phase - Träger für die Analyse der Methylester der langkettigen Fettsäuren ist Poly-, oder Di-äthylenglycolsuccinat (DEGS) oder Poly-, Diäthylenglycoladipat (DEGA) auf Kieselgur oder Chromosorb W, acid washed, 60/80 oder $80 / 100$ mesh. Die Konzentration der stationären Phase bewegt sich zwischen $7,5 \%$ bei Poly - und $15 \%$ bei Di-EGS oder EGA. Die Säulengröße beträgt meistens $5 \mathrm{ft} . \times 1 / 4$ in. oder $6 \mathrm{ft} . \times 1 / 4$ in., Arbeitstemperatur liegt zwischen $170^{\circ}$ und $200^{\circ}$; "angewandte Detektoken: "Argon ionisation"detector" oder F.I. D., flame ionisation detector. In der letzten Zeit wurden die besten Ergebnisse mit folgenden Parametern erreicht (12): 
Tab. 1

Vergleich der hier beschriebenen Methode der direkten elutionsfreien Methylierung (A) mit der Methylierung nach dem Verfahren von Doss und OETTE (B), gemessen an Serumlipidbestimmung

\begin{tabular}{|c|c|c|c|c|c|c|c|c|}
\hline \multirow{2}{*}{$\begin{array}{c}\text { Methylester }{ }^{2} \text { ) } \\
14: 0 \\
16: 0 \\
16: 1 \\
18: 0 \\
18: 1 \\
18: 2 \\
18: 3 \\
20: 3 \\
20: 4\end{array}$} & \multicolumn{2}{|c|}{$\begin{array}{l}\text { Freie Fettsäure } \\
\text { A }\end{array}$} & \multicolumn{4}{|c|}{$\begin{array}{l}\text { Fettsäuremethylester (\%) aus den Lipidgruppen: } \\
\text { Triglyceride } \\
\begin{array}{cccc}\text { A } & \text { Phospholipide }\end{array}\end{array}$} & \multicolumn{2}{|c|}{$\begin{array}{r}\text { Cholesterolester } \\
\text { A }\end{array}$} \\
\hline & $\begin{array}{r}1,6 \\
34,8 \\
4,1 \\
16,2 \\
32,0 \\
10,1 \\
1,2 \\
=\end{array}$ & $\begin{array}{r}1,5 \\
34,3 \\
4,1 \\
16,4 \\
32,3 \\
10,2 \\
1,2 \\
=\end{array}$ & $\begin{array}{r}1,7 \\
27,2 \\
5,6 \\
4,7 \\
44,5 \\
15,3 \\
1,0 \\
=\end{array}$ & $\begin{array}{r}1,8 \\
27,4 \\
5,8 \\
4,5 \\
44,3 \\
15,2 \\
1,0 \\
=\end{array}$ & $\begin{array}{r}\overline{30,7} \\
1,8 \\
15,8 \\
14,6 \\
23,7 \\
\overline{3,0} \\
10,4\end{array}$ & $\begin{array}{r}3 \overline{0,5} \\
1,8 \\
15,7 \\
14,7 \\
23,4 \\
\overline{3,2} \\
10,7\end{array}$ & $\begin{array}{r}\overline{13,2} \\
4,5 \\
1,8 \\
20,7 \\
52,8 \\
\overline{-} \\
\overline{7,0}\end{array}$ & $\begin{array}{r}\overline{13,1} \\
4,7 \\
1,9 \\
20,4 \\
53,0 \\
\overline{-}\end{array}$ \\
\hline $\begin{array}{l}\text { Gesamtmenge } \\
\text { der einzelnen } \\
\text { Lipidgruppen }\end{array}$ & 0,63 & 0,64 & ${ }_{m}^{68}$ & $n^{65}$ & $210_{n}$ & 208 & ${ }^{237}$ & 239 \\
\hline
\end{tabular}

2) Anzahl der C-Atome der Fettsäure : Anzahl der Doppelbindungen

$10 \mathrm{ft} . \times 1 / 16$ in. s. s., $3 \%$ TCEPE auf Aeropak 30, 80/100 mesh, Detektor: FID, Arbeitstemperatur: $150^{\circ}$

$2 \mathrm{ft} . \times 1 / 8$ in. s. s., $3 \%$ EGSS-X auf Aeropak 30, 80/100 mesh, Detektor: FID, programmierte Temperatur von $150^{\circ}$ bis $205^{\circ}$, $2 \%$ Min.

Bei dieser Arbeit wurde benutzt:

$5 \mathrm{ft} . \times 1 / 4$ in. s. s., $15 \%$ DEGS auf Chromosorb W 80/100, acid washed; Arbeitstemperatur: $190^{\circ}$, Detektor: FID Durchflußgeschwindigkeit des Trägergases: $25 \mathrm{ml} / \mathrm{Min}$., $\mathrm{N}_{2}$.

\section{Ergebnisse}

In der vorliegenden Tabelle sind neben den Ergebnissen, die durch die hier beschriebene Methode gewonnen wurden (unter „ „A") auch die Ergebnisse, die nach der Methode von Doss und OETTE (7) erzielt wurden (unter „B“'), aufgeführt. Um die alkalische Umesterung nach Doss und OETTE durchzuführen, wurden vorher die Phospholipide nach der Veröffentlichung von ZAHLER (10) eluiert, Elution der übrigen Lipidfraktionen erfolgte durch die Behandlung des Kieselgels mit Dichlormethan. Dabei wurden die freien Fettsăuren, nach der Verdampfung der Elutionsmittel, durch die Behandlung mit $\mathrm{BF}_{3}$-Methanol-Lösung nach MeTCALFE und ScHMrtz (13) verestert. Die in der Tabelle angegebenen Werte sind Mittelwerte aus 10 Bestimmungen ein und desselben normalen menschlichen Serums; dabei wurden $5 \mathrm{Be}-$ stimmungen mit der hier beschriebenen direkten Methylierung und 5 durch die Methode von Doss und Oetre (7) gewonnen. $\mathrm{Da}$ bei den Ergebnissen von Doss und OETTE die alkalisch-katalysierte Umesterung schon mit der durch methanolische $\mathrm{HCl}$ (14) säure-katalysierten verglichen wurde, erübrigte sich in unserer Arbeit die nochmalige Durchführung dieser Analyse. Wie aus der Tabelle und aus der Arbeit von Doss und Oetre (7) ersichtlich ist, sind die Ergebnisse der drei erwähnten Methoden in etwa gleich.

Die Umrechnung des Gewichtes der gaschromatographisch bestimmten Fettsäuremethylester auf die Quantität der einzelnen Lipidfraktionen gründet sich an der Bestimmung der durchschnittlichen Molekulargewichtes $\bar{M}$ der Methylester der jeweiligen Lipidfraktion. Die Analyse des menschlichen Normalserums ergibt unter Berücksichtigung der prozentualen Vertretung der einzelnen Fettsäuren in der Lipidfraktion folgende Werte von $\bar{M}:$ Freie Fettsäuren $=286$, Triglyceride $\doteq 287$,
Phospholipide $=291$, Cholesterolester $=292$. Man sieht, daß sich die einzelnen Werte des durchschnittlichen Molekulargewichtes $\bar{M}$, trotz der verschiedenen Vertretung der einzelnen Fettsäuren in jeder Lipidfraktion, voneinander kaum unterscheiden. Aus diesem Grunde können wir, ohne große Fehler zu machen, für jede Lipidfraktion denselben Wert von $\bar{M}$ annehmen: $\bar{M}=$ 290.

Die Umrechnung der gaschromatographisch bestimmten Menge der Fettsäuremethylester (ME) sieht unter Annahme $\bar{M}=290$ folgendermaßen aus:

Triglyceride (mg) = ME (mg)

Freie Fettsäuren $(\mathrm{mVal})=\mathrm{ME}(\mathrm{mg}): 290$

Cholesterolester $(\mathrm{mg})=\mathrm{ME}(\mathrm{mg}) \cdot 2,22$

Phospholipide (mg) $=\mathrm{ME}(\mathrm{mg}) \cdot 1,95$

Bemerkung: Der Koeffizient 1,95 bei der PhospholipidFraktion bedarf noch weiterer Erläuterung. Aus der Arbeit von ZaHLER (10) geht folgende prozentuale Vertretung der einzelnen Phosphatide in der Phospholipid-Fraktion hervor: Kephalin 2,1\%; Lecithin 58,6\%; Lysolecithin 9,2\%; Sphingomyelin 17,5\%; die restlichen $12,6 \%$ bilden weitere, nicht näher bestimmte Phosphatide. Die alkalische Umesterung transferiert nur die ersten drei Phosphatide in die Methylesterform, während Sphingomyelin und die übrigen Phosphatide, also zusammen 30\% der Phospholipid-Fraktion, sich nicht umestern lassen (siehe Diskussion). Der Umrechnungskoeffizient der Methylester in bezug auf Kephalin, Lecithin und Lysolecithin ist unter Berücksichtigung der prozentualen Vertretung in der PhospholipidFraktion des menschlichen Serums gleich 1,36. Da aber die drei erwähnten Phosphatide zusammen nur $70 \%$ der Gesamtfraktion bilden, muß eine weitere Korrektur durchgeführt werden: $1,36 \cdot \frac{100}{70}$ ergibt dann 1,95 .

\section{Diskussion}

Die hier beschriebene Methodik wirft folgende Fragen auf: Wie weit beeinflußt die Menge und Art des abgesaugten Kieselgels die Effektivität der Methylierung und somit die quantitative Bestimmung? Inwiefern muß die bei Doss und OEtTE (7) aufgeführte Bedingung, $\mathrm{da} ß$ die Lipide vor der Zugabe des $\mathrm{Na}$-Methylats in dem Lösungsmittel vollständig gelöst werden müssen, 
erfüllt sein? Wie beeinflußt die in dem dünnschichtchromatographischen Fließmittelsystem enthalteneEssigsäure die alkalische Hydrolyse der Phospholipide?

Unter Erwägung dieser Fragen erscheint uns die hier angegebene Methodik im Rahmen der gegenwärtig angewandten dünnschichtchromatographischen Verfahren universell geeignet zu sein. Nur eine extrem große Menge von Kieselgel, das volumenmäßig etwa der Hälfte aller zugegebenen flüssigen Reagenzien entspricht, beeinflußt die quantitative Bestimmung ungünstig; das gilt besonders bei der am schwierigsten hydrolysierbaten Cholesterolester-Fraktion. Eine so große Menge von Kieselgel kommt allerdings in der Praxis kaum vor. Auch bei der $2 \mathrm{~mm}$ dicken Schicht der „gestreuten" Platten war eine quantitative Methylierung erzielt.

Die Ergebnisse der quantitativen Bestimmung werden auch von der Korngröße des Kieselgels nicht beeinträchtigt. In hier durchgeführten Bestimmungen wurden Korngrößen von $10-200 \mu \mathrm{m}$ benutzt.

Ebenfalls die Zugabe von Gips zum Kieselgel oder die Anwendung des Dichlorfluoresceins als Detektor der Flecke haben die Bestimmung nicht beeinflußt.

Auch bei folgenden Mengen der Lipidfraktionen wurde eine quantitative Umesterung erreicht: $6 \mathrm{mg}$ Phospholipide, $12 \mathrm{mg}$ Triglyceride, $6 \mathrm{mg}$ Cholesterolester und $3 \mathrm{mg}$ Freie Fettsäuren. Andererseits wurden auch 1000fach niedrigere Mengen erfolgreich analysiert.

Die Anwendung des wäßr. Na-Methylats (methanolische $\mathrm{NaOH}$ ) führte zum selben Ergebnis wie die Anwendung des wasserfreien Reagenz. Kleine Mengen von Wasser wirken in diesem Falle also nicht störend (15).

Die Reihenfolge der Zugabe der Reagenzien zum Kieselgel vor der Methylierung hat keinen Einfluß auf die Ergebnisse, es ist also nicht unbedingt notwendig, die Lipide vorber im Lösungsmittel vollständig zu lösen. Dagegen ist es erforderlich, die Reihenfolge der Reagenzien nach der Methylation genau einzuhalten: 1. Petroläther, 2. wäßr. $\mathrm{HCl}$. Wenn zunächst $\mathrm{HCl}$ zugegeben wurde, hat sich die Ausbeute der Analyse auf $60-80 \%$ verringert.

Die Acetonzugabe diente einer besseren Mischung von Petroläther und Na-Methylat. Ohne diese Zugabe bildete sich ein Zweiphasen-System und die Umesterung verlief nicht ganz vollständig (etwa 90-100\%). Falls größete
Mengen von Aceton oder Petroläther zugegeben wurden oder eine konzentriertere Lösung von Na-Methylat, z. B. $1 \mathrm{~N}$, angewandt wurde, führte das besonders bei der Fraktion der Cholesterolester zú einer Verminderung der Ausbeute bis auf 70\%. Ebenfalls führt die Anwendung einer weniger konzentrierten Lösung des $\mathrm{Na}$ Methylats zu keinen befriedigenden Ergebnissen, so daß nur die $0,5 \mathrm{~N}$-Lösung optimal und empfehlenswert ist.

Der 1 proz. Anteil der Essigsäure im dünnschichtchromatographischen Fließmittelsystem beeinflußt durch seinen niedrigen $\mathrm{pH}$-Wert nur die unterste Fraktion auf der Platte, die Phospholipide. Ein Teil des Methylats wird dadurch neutralisiert und das. macht eine Zugabe von $5 \mathrm{~m} l \mathrm{Na}$-Methylat bei dieser Fraktion, statt $4 \mathrm{~m} l$ wie bei den anderen Fraktionen, erforderlich. Diese Menge reicht bei einem Gehalt bis zu $1 \mathrm{mVal}$ Essigsäure $(0,06 \mathrm{~m} / \mathrm{im}$ abgesaugten Kieselgel aus. Bei Fließmittelsystemen mit einem höheren Säureanteil müßte man entsprechend mehr Na-Methylat zugeben.

Ähnlich wie bei allen Methoden, die sich der alkalischen Umesterung, bedienen, führt auch die bier beschriebene Methode nicht zur Bildung von Methylestern bei solchen Fettsäuren, die an das ursprüngliche Molekül amidartig gebunden sind. Das betrifft z. B. Sphingomyelin, wo die Fettsäure mit dem Aminodiol Sphingosin durch eine Amidbindung verbunden ist. Bei der Analyse der Phospholipid-Fraktion fehlen also die Methylester der Fettsäuren, die an Sphingomyelin gebunden sind. Da im Mittelpunkt des Interesses vor allem die ungesättigten, besonders die essentiellen Fettsäuren stehen, wird dies nicht besonders stören, $\mathrm{da}$ an Sphingomyelin fast ausschließlich gesättigte Fettsäuren gebunden sind (16).

Der einzige Nachteil der hier beschriebenen Methodik im Vergleich mit der Methode nach Doss und OETrE (7) besteht in der Notwendigkeit, doppelt soviel Reagenzgläser zu benutzen.

Dagegen benötigt diese Methode keine besondere Apparatur und erheblich weniger Reagenzien, als sonst zur Elution der Lipidgruppen nötig sind. Die Verkürzung der Analysenzeit von der Auftragung der Lipide auf die dünnschichtchromatographische Platte bis zur Gaschromatographie der Methylester auf nur 1-2 Stdn. ist der besonders große Vorteil des hier angegebenen Verfahrens.

\section{Literatur}

1. Kuksis, A., L. Marai und D. A. Gornall, J. Lipid Res. 8 , 352 (1967). - 2. Bauman, F., A. Klein und J. Gill, Varian Aerograph (1967). - 3. Brezenskr, J. J., Federation Proc. 23, 503 (1964). - 4. De Bohner, L. S., E. F. Soto und T. DE Cohan, J. Chromatog. 17, 513 (1965). - 5. Arramson, D. und M. Blecher, J. Lipid Res. 5, 628 (1964). - 6. Luddy, F. E., R. A BARFord und R. W. RIEMENSChNEIDER, J. Amer. Oil Chemist's Soc. 37, 447 (1960). - 7. Doss, M. und K. Oetre, diese Z. 3, 125 (1965). - 8. Folch, J., M. Less und G. H. Sioane Stanlex, J. biol. Chemistry 226, 497 (1957). - 9. RenKoneN, O., T. U.
Kosunen und O. V. Renkonen, Ann. med. exper. biol. Fenniae 41, 375 (1963). - 10. ZAFLER, P., diese Z. 5, 191 (1967). 11. Schlenk, H. und J. L. GellermanN, Analytic. Chem. 32, 1412 (1960). - 12. Hammarstrand, K., Varian Aerograph (1966). 13. Metcalfe, L. D. und A. A. Schmitz, Analytic. Chem. 33, 363 (1961). - 14. Stofrel, W., F. Chu und E. H. Afrrens, Jr., Analytic. Chem. 31, 307 (1959). - 15. Kuemmen, D. F., J. Amer. Oil Chemists' Soc. 35, 41 (1958). - 16. SweELeY, C. C., J. Lipid Res. 4, 402 (1963).

Dipl.:Ing. Petr Hušek z. Zt. Forschungsinstitut Borstel D-2061 Borstel 